http://dx.doi.org/10.18778/2196-8403.2019.07

KRISTIN EICHHORN

\title{
Der revolutionäre Dichter. JOHANNES R. BECHERS ästhetische Rezeption der Russischen Revolution
}

Der Artikel untersucht die zwei Phasen der ästhetischen Rezeption der Russischen Revolution bei JoHANNES R. BECHER anhand der zwei Fassungen des Dramas Arbeiter Bauern Soldaten von 1919 bzw. 1924. In der ersten Phase, BECHERS unmittelbarer Revolutionsbegeisterung, tritt die kommunistische Ideologie an die Seite des expressionistischen Aufbruchspathos, dem sie eine Richtung verleiht. In der zweiten Phase nimmt die Auseinandersetzung mit der Russischen Revolution paradoxerweise stark autobiographische Züge an - obwohl BECHER gleichzeitig das Moment des Kollektivismus argumentativ stärkt.

The Aesthetic Reception of the Russian Revolution in the Works of JoHANNES R. BECHER

The article examines two phases in JOHANNES R. BECHER's response to the Russian Revolution and its aesthetic consequences, by looking at the two versions of his play Arbeiter Bauern Soldaten (Workers Peasants Soldiers; 1919/1924). In the first phase, BECHER expresses his enthusiasm for the revolution and combines communist ideology with the expressionistic pathos of awakening into a new and groundbreaking future. In the second phase, the topic of the Russian Revolution is, paradoxically, more autobiographicallyshaped, and it is accompanied by stronger arguments in support of collectivism.

Estetyczna recepcja Rewolucji Październikowej u JoHAnnesa R. BeChERA Artykuł analizuje dwie fazy recepcji Rewolucji Październikowej w twórczości JOHANNESA R. Bechera. Podstawą są dwie wersje dramatu Arbeiter Bauern Soldaten, pierwsza z roku 1919, druga z roku 1924. Okres pierwszy charakteryzuje się w światopoglądzie BECHERA rewolucyjnym entuzjazmem. Wówczas ideologii komunistycznej wtóruje charakterystyczny dla ekspresjonizmu patos przebudzenia i przełomu. Kilka lat później można dostrzec, że tematowi Rewolucji Październikowej paradoksalnie towarzyszą konotacje autobiograficzne przy jednoczesnym wzmocnieniu argumentacji na rzecz procesu kolektywizacji. 
In gewisser Weise ist JOHANNES R. BECHER der deutsche Dichter der Oktoberrevolution. Die wenigsten deutschen Autoren haben ,die Revolution so stürmisch begrüßt und weiterzutreiben versucht“ (GW II:572). Schon aus dem Jahr 1917 ist ein handschriftliches „Widmungsblatt an die Russische Revolution“ überliefert, das BECHER auf den 13. Oktober datierte (BECHER 1959:116). Bekannter ist das 1919 erschienene Gedicht „Gruß des deutschen Dichters an die Russische Föderative Sowjet=Republik“, das die Begeisterung BECHERS für die Revolution mustergültig auf den Punkt bringt und deshalb als eines der frühesten Bekenntnisse zu den Ereignissen in Russland gilt (GW II:18f.). ${ }^{1}$ Ansonsten ist noch auf das 1931 auf die Bühne gebrachte, Epos des sozialistischen Aufbaus' Der Große Plan hingewiesen worden (vgl. REUS 1978:131-144).

Einerseits geht BECHERS literarische Beschäftigung mit der Oktoberrevolution weit über die genannten Einzeltexte hinaus, auf die das Augenmerk der Forschung bislang gerichtet war. ${ }^{2}$ Andererseits stecken hinter den genannten Werken auch zwei getrennte Rezeptionsphasen, die mit dem politischen Interesse BECHERS korrelieren. Er reagierte zunächst direkt nach den revolutionären Ereignisse 1918/1919 mit einer Reihe von Dichtungen: Vom Ton der subjektiven Liebeslyrik fast überdeckte Spuren der Rezeption finden sich in den Gedichten um Lotte (1919), deren Entstehung bis in den Oktober 1917 zurückreicht. ${ }^{3}$ Der „Gruß des deutschen Dichters“ steht in einem Kontext mehrerer literarischer Arbeiten aus dem Jahr 1919, die sämtlich Bezüge zu den beiden Revolutionen aufweisen - allen voran der Lyrikband An Alle!, der das Gedicht enthält (vgl. BECHER 1919). Nach dieser ersten Rezeptionsphase wandte sich BECHER für einige Jahre von der politischen Literatur ab, zugunsten religiöser Einkehr und mystisch inspirierter Dichtung. ${ }^{4}$ Mit dem KPD-Eintritt 1923 änderte sich BECHERS ästhetisches Programm erneut und in radikalerer Weise. Infolge dieses Schritts begann seine zweite Beschäftigung mit der Oktoberrevolution, zu der neben Der Große Plan u. a. das Grabgedicht auf Lenin (vgl. BECHER 1924) und der Giftgasroman $(\mathrm{CHCl}=\mathrm{CH})_{3} \mathrm{As}$ (Levisite) oder Der einzig gerechte Krieg (1926) gehören (BECHER 1926a).

Beide Phasen lassen sich besonders gut anhand des Dramas Arbeiter Bauern Soldaten-nachvollziehen, das BECHER 1919 schrieb und 1924 in überarbeiteter

\footnotetext{
1 Vgl. dazu kritisch WALTER (1978:618).

2 Am differenziertesten macht noch auf diese Kontexte aufmerksam SCHLENSTEDT (1976:14-52; 113-158).

3 Vgl. dazu Schlenstedt (1976:14ff.).

4 Vgl. etwa die Gedichtbände Ewig im Aufruhr (1920), Zion (1920), Der Gestorbene (1921), Um Gott (1921), Verklärung (1922).
} 
Fassung präsentierte. Die Erkenntnisse, die sich aus diesem Vergleich gewinnen lassen, sind aufschlussreich für die deutsche Rezeption der Russischen Revolution von 1917 als Ganzes. Abgesehen von ihrer Datierung - BECHERS ,Rückkehr zur Politik erfolgt nicht von ungefähr in einer Zeit, als sich die politischen Gegensätze in der Weimarer Republik zuspitzen ${ }^{5}$ - zeigen sie auf, inwieweit man in Deutschland zu neuen künstlerischen Mitteln greift, für die es in Russland Vorbilder gibt. Vor allem aber lässt sich eine unterschiedlich intensive Auseinandersetzung mit der kommunistischen Lehre beobachten, die in die eine Neufassung des Dichterbegriffs mündet, an dem BECHER entgegen den neusachlichen Tendenzen in seinem Werk weiter festhält (vgl. BECKER 2009:256).

Im ersten Rezeptionsgang fungiert das revolutionäre Moment als reine Konkretisierung des expressionistischen Aufbruchspathos, das den Dichter als kommunistischen Propheten in der Tradition des poeta vates versteht. Demgegenüber kommt es Mitte der 1920er Jahre zu einem sehr viel mehr distinkten, autobiographisch fundierten und - im Sinne des Marxismus - stark antibürgerlichen Modell. An die Stelle des abstrakten genialen Individuums, das die Massen anführt, tritt nun die eigene Biographie, die in einem Vorbildcharakter zur Nachahmung aufruft, aber auch als Nachweis des gesellschaftlichen Wandels genutzt wird - was paradoxerweise bedeutet, dass der Ruf nach Kollektivierung zu einer Zunahme an autobiographisch fundierten Texten führt, weil der bürgerliche Schriftsteller seinen Platz in der proletarischen Bewegung erst finden und verteidigen muss. ${ }^{6}$

\section{Die erste Phase der Revolutionsrezeption: Arbeiter Bauern Soldaten. Der Aufbruch eines Volkes zu Gott. Ein Festspiel (1919)}

Schon in seinem expressionistischen Hauptwerk Verfall und Triumph (1914) rief BECHER mehrfach zu einem Ausbruch aus den dekadenten bürgerlichen Verhältnissen des Kaiserreichs (=,Verfall' ${ }^{\circ}$ ) auf, ohne dass so recht klar war, welche Gestalt der sich daran anschließende ,Triumph ‘ haben sollte. ${ }^{7}$ Vor diesem Hintergrund erschienen ihm die gesellschaftlichen Ereignisse von 1917/1918 als Bestätigung der expressionistischen Prognose, die gleichzeitig eine inhaltliche

5 Zum literarischen Niederschlag im Einzelnen vgl. KIESEL (2017).

$6 \quad$ Als eine beliebte Legitimationsstrategie weist man den bürgerlichen Künstlern die Rolle von ,Geburtshelfern' zu, die die ästhetische Produktion des Proletariats in Gang zu setzen helfen (vgl. FÄHNDERS 2010:252).

7 Vgl. zu dieser - für den Frühexpressionismus nicht untypischen - Vagheit der entworfenen Utopie BEHRENS (2003:52). 
Konkretisierung des erst ex negativo entworfenen Zukunftsideals versprechen. Es ist diese ,Passform' der Oktoberrevolution, die BECHERS Identifikation mit Sowjet-Russland initiiert, nicht so sehr eine intellektuelle Durchdringung der marxistischen Lehre, die ihr zugrunde liegt (vgl. GW II:573f.). Auch ist es nicht eigentlich ein politisches Programm, das ihn mit den kommunistischen Ideen sympathisieren lässt, wenngleich sich sozialkritische Themen schon seit 1914 durch sein Werk ziehen. ${ }^{8}$ Er zeigte sich primär von der ästhetischen Aufbruchsstimmung angeregt, die nach dem Ersten Weltkrieg in Deutschland einsetzte, und sah mit der Oktoberrevolution den Zeitpunkt gekommen, sein bisheriges künstlerisches Verfahren zu überdenken und durch ein ,zeitgemäßeres` zu ersetzen. ${ }^{9}$

Besonders augenscheinlich wird der ästhetische Einfluss der Revolutionserfahrung daran, dass BECHER, der bis dahin vor allem als Lyriker und Verfasser von (kurzer) Erzählprosa aufgetreten war, sich 1918/1919 erstmals dem Drama zuwandte. Er befindet sich damit in guter Gesellschaft, denn auch Autoren wie Franz Jung, Erich Mühsam oder Karl August Wittvogel entdeckten das Theater infolge der revolutionären Ereignisse als Instrument, ,das ihren aktivistischen, manifestbereiten Ideen in besonderer Weise entspricht" (NÖSSIG / ROSENBERG / SCHRADER 1980:121). Das früheste von BECHER überlieferte Stück ist das ,dramatische Gedicht' Ikaros, das 1919 im von BECHERS Freund Alfred Wolfenstein herausgegebenen Jahrbuch Erhebung erschien (GW VIII:5-15).

Intensiver wird die Auseinandersetzung mit der Revolution mit dem Stück Arbeiter Bauern Soldaten, bei dem sich BECHER erkennbar von den jüngsten theatralischen Entwicklungen anregen ließ. Nachdem anfangs der traditionalistische Ansatz Max Reinhardts die Berliner Bühnen dominiert hatte, bemühten sich in Deutschland vor allem Karlheinz Martin und Rudolf Leonhard um die Etablierung einer politischen Bühne - zunächst über die Gründung der kurze Zeit später von ihnen wieder verlassenen „Tribüne“, dann über das „Proletarische Theater", das ebenso heißt wie das spätere, aber davon unabhängige Piscator-

8 Entsprechend war es leicht, in den Dichtungen des expressionistischen Jahrzehnts Vorzeichen von BECHERS späterer Entwicklung auszumachen (vgl. HOPSTER 1969:102).

9 Aus dieser - nicht zuletzt ästhetischen - Motivation ergeben sich die zahlreichen stilistischen Umbrüche im Gesamtwerk, die BECHER zunächst immer zu überdecken versuchte, bis er sie schließlich im Spätwerk als konstitutives Moment zum Thema machte. Vgl. vor allem das letzte Romanfragment mit dem programmatischen Titel Wiederanders (GW XI:435-607). 
Unternehmen. ${ }^{10}$ Die russischen ästhetischen Entwicklungen im Rahmen von Agitprop und Proletkult waren für die deutschen Künstler ,zunächst unter politischen und sozialen Gesichtspunkten ihrer eigenen künstlerischen Existenz" von Belang und wurden unter dem Postulat eines klaren „Führungsanspruch[s] der Künstler im revolutionären Kampf des Proletariats" aufgegriffen (NÖSSIG / ROSENBERG / SCHRADER 1980:125). Entsprechend lässt sich in BECHERS Arbeiter Bauern Soldaten eine unübersehbare Kontinuität nicht nur zum Expressionismus, sondern auch zum Autorschaftsmodell des poeta vates ausmachen, der nun als eine Art kommunistischer Prophet agiert.

Bei der Erstfassung aus dem Jahr 1919 handelt es sich laut Gattungsangabe auf dem Titelblatt um ein ,Festspiel', weshalb sich in der Forschung der Begriff ,Festspielfassung' eingebürgert hat (vgl. HARTUNG 2002:283-292), um die erste Version von der späteren Überarbeitung von 1924 zu unterscheiden, die BECHER als „Entwurf zu einem revolutionären Kampfdrama“ bezeichnet (GW VIII:101). Was gegenüber Ikaros (und seinen späteren Dramen aus den 1940er und 1950er Jahren) auffällt, ist das Fehlen individueller Figuren. Stattdessen arbeitet BECHER in Arbeiter Bauern Soldaten - wie auch später in Der Große Plan - mit „Stimmen“, die hier auf Typen wie „Der Mann“, „Die Frau“ oder „Der Gewaltherr“ verteilt werden (GW VIII:22). Eine nacherzählbare Handlung gibt es nur in Ansätzen; das Stück hat insgesamt eher argumentativen als szenischen Charakter. Nachdem im ersten Teil von „Mann“ und „Frau“ der Aufstand der Unterdrückten gegen ihren Herrn beschworen wird, ruft im mittleren Teil der Gewaltherr seine Truppen zusammen, um sich gegen den Aufstand zur Wehr zu setzen. Die Frau überzeugt zunächst den Gewaltherrn, seine Ämter niederzulegen. Als dieser indes bemerkt, dass er noch Unterstützer hat, befiehlt er, die Frau zu töten, worauf er vom Mob gerichtet wird. Der dritte Teil kehrt zum beschwörenden Ton zurück: Arbeiter, Bauern und Soldaten marschieren einer besseren Zukunft entgegen; der Mann, der seine Funktion erfüllt hat, wirft seine menschliche Gestalt ab und vergeistigt sich wie schon die Frau zur „Stimme [...] im Licht“ (GW VIII:85).

Die Bezüge zu den Revolutionen von 1917/1918 sind, wie leicht zu erkennen ist, äußerst reduziert, weil die wenigen konkreteren Anspielungen auf die Realität durch die alttestamentarische und allegorische Anlage überdeckt werden: Hinter dem Mann und der Frau stehen eigentlich Karl Liebknecht und Rosa Luxemburg, was freilich erst die Kampfdrama-Fassung von 1924 explizit macht. Die

10 Eigenständige Forschungen zum Unternehmen von Martin und Leonhard gibt es nicht. Siehe daher exemplarisch das Referat im Kontext der Piscator-Bühne bei PATTERSON (1981:113f.). 
Festspielfassung hingegen markiert diesen Bezug allein über die alternative Bezeichnung der Frau als „Jüdin“ (GW VIII:48) und die entscheidende Initiatorenrolle, die sie für die Revolution spielt.

Ähnlich unbestimmt reagiert BECHER auch in formaler Hinsicht auf die Revolution. Gerade die der Festspielfassung von Arbeiter Bauern Soldaten vorangestellte „Vorbereitung" (GW VIII:19) macht mit ihrem sprachexperimentellen Duktus mehr als deutlich, wie stark die revolutionären Ereignisse von 1917 und 1918 das expressionistische Aufbruchspathos fortsetzen. Rhetorisch wird sichtbar, dass ein Aufstand des Proletariats gemeint ist, wenn die „Ärmsten [...] von den Bänken“ (GW VIII:19) springen und sich zur Massenbewegung zusammenschließen; diese Passagen gehen indes in den wesentlich vageren Aufrufen unter, die noch kaum eine intellektuelle Auseinandersetzung mit den Grundlagen der Revolution als solche erkennen lassen:

Mensch schmeiß empor dich! Steig! Entflamm!

Zerdolch dich Mensch! Verreck! Verschlamm!

Beiß! Spritze vor! Granit! Und Krallen!

Einst sollst du stehn! Noch mußt du fallen!

Rott aus! Stirb ab! Ras auf! Feg hin! --

... Du: Märchen-Lamm! Und Hüterin!

Du innerste, die heilige Säule.

Wir noch Verrat, gestopft mit Fäule.

Die Mörder gehen ab wie Schleim.

Doch in uns wurzelt Stern. Der keimt.

Die Ärmsten springen von den Bänken.

Jetzt Führer auf! Laß Massen schwenken!

Brennt Barikaden! Plätze knallen.

(GW VIII:19)

Fließend ist schließlich der Übergang von einer religiösen Heilserwartung zur kommunistischen Friedensutopie. Schon in Verfall und Triumph schwankt BECHER zwischen einer Beschwörung des christlichen Gottes und der Überlegung, dass Religion als ,Opium fürs Volk' eine Beseitigung sozialer Missstände verhindert, weil sich die Betroffenen mit ihrem Schicksal abgefunden haben (BECHER 1914:32f.). Entsprechend hat BECHER in An Alle! kein Problem damit, das „Heer // Der proletarischen Nation“ mit „Gottes Geist“ zu identifizieren und die Gleichheit aller („Und keiner wird da über den anderen schalten!") sowohl mit dem christlichen Paradies als auch mit dem Entwurf einer nachrevolutionären Gesellschaftsordnung in Verbindung zu bringen (GW II:16). 
Darüber hinaus versucht BECHER in formaler Hinsicht, die Berichte über die russischen Theaterentwicklungen mit einem ,klassischen“ Theatermodell zu versöhnen, um die Kunst gegenüber der reinen Propaganda zu retten (ANONYMUS 1973). Deswegen verbindet er das Konzept der Aufhebung der Grenze zwischen Zuschauer und Publikum mit der Tradition des Festspiels nach antikem Muster, das das Schauspiel in eine kultische Feier überführt, so wie im Agitprop nach dem Stück gemeinsam die Internationale angestimmt und zur Revolution aufgerufen wird (vgl. INNES 1972:25).

Damit werden Charakteristika der stark rudimentären russischen ,Stücke', die mehr oder weniger aus der Notwendigkeit heraus entstanden waren, nun als ästhetisch motivierte Entscheidungen rezipiert. Hatte das Agitproptheater etwa „auf den Mangel an dramatischem Spielmaterial“ mit Sprechchören reagiert (REUS 1978:52), greift BECHER das Moment bereits als konstitutiv für das revolutionäre Theater als solches auf und baut es deshalb gezielt in seine ,Dramenhandlung“ ein. Durch die Arbeit mit „Stimmen“ anstelle von individuellen Charakteren kommt eine Konstellation zustande, in der einzelne Sprecher Zweifel und Probleme ansprechen, bevor Gegenstimmen eine hoffnungsvollere Alternative in Aussicht stellen. In den meisten Fällen jedoch nutzt BECHER das Mittel des Chors, um den Zusammenschluss Einzelner zu einer gemeinsamen Bewegung deutlich zu machen. Nachdem ein Schläfer, ein Toter und ein stummer Blinder ihre Positionen dargelegt haben, baut sich nach und nach eine Mehrzahl auf (,,einige der Schläfer“, „,einige der Toten“, „einige der stummen Blinden“; GW VIII:26), bis die drei Gruppen als eigene Chöre auftreten (GW VIII:27). ${ }^{11}$

Das Prinzip des ,Vom Ich zum Wir' bildet auch die entscheidende Grundlage dafür, wie die Festspielfassung von 1919 schließlich das Publikum involviert - ein wichtiges Element im proletarisch-revolutionären Theater, das agitieren und Teil der revolutionären Bewegung sein will (vgl. WOLFF 1985). Der „Aufbruch der Zuschauer" (GW VIII:95), der auf den zahlreicher anderer Gruppen folgt, die das Drama vorgestellt hat, bildet den Abschluss des dritten Teils und somit gewissermaßen das Ergebnis des Stücks. Wieder kommt erst ein einzelner Zuschauer (als Figur des Stücks) zu Wort, das in ein „Gemurmel unter den Zuschauern" übergeht (GW VIII:95). Am Ende schließen sich alle Gruppen einschließlich der Zuschauer zum kollektiven Aufruf zusammen, der nun ,,von allen Seiten“ erschallt: „Auf! Auf! Auf! Ins Land der Verheißung! / Ins Land der Verheißung. Ins heilige Land...“(GW VIII:99).

11 Dieses Verfahren wird in der Zweitfassung ausgebaut und auf die ,Titelhelden“ Arbeiter, Bauern und Soldaten übertragen (vgl. GW VIII:118-120). 
Die politische Wirkung des Stücks ergibt sich also in seiner Erstfassung intentional dadurch, dass ein Einzelner seine Stimme erhebt und die Notwendigkeit zum revolutionären Aufbruch verdeutlicht, wie dies auch BECHERS Stück letztendlich tut. Die Relevanz des Dichters ergibt sich dabei aus der Bedeutung, die Lieder und Gesänge für den revolutionären Prozess haben. ${ }^{12}$ Erst die Versform ermöglicht jenen Gleichschritt, der für eine Massenbewegung konstitutiv ist; folglich braucht es Dichter und nicht nur (Prosa-)Schriftsteller, die die Verse für den politischen Prozess vorgeben. Bei allem postulierten Kollektivismus wird der Massengesang in Arbeiter Bauern Soldaten somit performativ aus individuellen Stimmen zusammengesetzt und bedarf der Initialleistung und ,Führerschaft' von Einzelpersonen - Mann und Frau, um die Revolution in Gang zu bringen, indem sie die Stichworte vorgeben, die dann aus dem Munde aller wiedertönen. ${ }^{13}$ Eben aus diesem Grund ist es ausgerechnet der deutsche Dichter, der im Gruß-Gedicht das Wort an die Sowjetrepublik richtet und das ,heilige Reich“ bzw. das „Paradies“ verkündet, das auf die ,Zertrümmerung“ der „westliche[n] Demokratien“ folgen wird (GW II:19).

\section{Die zweite Phase der Revolutionsrezeption: Arbeiter Bauern Soldaten. Entwurf zu einem revolutionären Kampfdrama (1924)}

Erst die zweite Auseinandersetzung mit dem Kommunismus und der revolutionären Aufgabe der Literatur führt zu einer nachhaltigen Änderung von BECHERS Selbstverständnis als Dichter. Hatte er 1918/1919 versucht, das utopische Potential des Kommunismus mit den Schreibweisen zu verbinden, die er vorher vertreten hatte, markiert der Eintritt in die KPD 1923 einen radikalen Bruch mit dem Frühwerk, indem sich BECHER nun vollständig mit der revolutionären Bewegung identifiziert und seine Rolle in ihr zu bestimmen sucht. Damit verbunden ist zunächst vor allem das Bekenntnis zum Atheismus, das den wesentlichen Ausschlagpunkt für Überarbeitung von Arbeiter Bauern Soldaten gibt. In Übereinstimmung mit Lenin betrachtet BECHER nun „den Glauben an Gott“ als „unvereinbar mit der Mitgliedschaft in der revolutionären Partei“ (RYKLIN 2008:13) und zeichnet das Vertrauen auf eine übernatürliche Macht entsprechend der bolschewistischen Weltsicht als Illusion und als gefährliche Krankheit: „Immer

12 Zur deutschen Perspektive HAGELWEIDE (1968).

13 Vgl. vor allem schon das Ende des ersten Teils mit dem ,„platzende[n] Aufruf des Mannes“, der bezeichnenderweise ,Arbeiter Bauern Soldaten!“ lautet (GW VIII:42). 
wieder, verstrickt in ein Traum-Dickicht, lechzt du nach Gott... Und diese Seuche, die Gott heißt, sie rafft dich dahin“ (GW VIII:149).

In Arbeiter Bauern Soldaten werden entsprechende Elemente folglich in der Überarbeitung ,radikal ausgemerzt“" (GW VIII:104). Stattdessen präzisiert BECHER die revolutionäre Absicht seines nunmehr historisch klarer zu verortenden Stücks, dessen Handlung inzwischen nicht mehr die unmittelbare Gegenwart betrifft, sondern eine halbe Dekade zurückblickt. War die Festspielfassung zeitlich nicht konkret zu verorten, setzt die ,Handlung ' in der neuen Version im Ersten Weltkrieg ein: „Vier Jahre wohl nun hocken wir schon tief in den Gräben“ (GW VIII:127). Wo im Dramentext von 1919 nur abstrakt von einer Jüdin die Rede ist, in der mit entsprechendem Kontextwissen Rosa Luxemburg als Vorbild zu erkennen ist, fallen jetzt die Namen „Karl, Rosa, Radek“ (GW VIII:141) und der Tod der „Führer“ (GW VIII:138) wird durch die Nennung des Landwehrkanals und durch die Anspielung auf den Kieler Matrosenaufstand historisch eindeutig (GW VIII:137 bzw. 138).

Noch in einer anderen Hinsicht wird BECHER konkreter: Er nutzt das Stück, das von Anfang an die Oktoberrevolution als Vorbild für Deutschland auffasste, 1924 für eine direktere Gegenüberstellung der deutschen und der russischen Verhältnisse, die nach den revolutionären Ereignissen beide vom Kaisertum zur Volksherrschaft übergingen - allerdings auf denkbar unterschiedliche Weise. Mit den Mitteln von Satire und Groteske prangert BECHER die aus Sicht der KPD häufig kritisierten konterrevolutionären Fehlentwicklungen in der Weimarer Republik an, die sich als demokratisches System nicht mit der Sowjetunion messen kann. Während der Gruß des deutschen Dichters 1919 noch die Zuversicht ausdrückt, dass eine Revolution nach russischem Vorbild auch auf deutschem Boden in Kürze erfolgreich sein wird, gibt es nun ein politisches System, das als Scheindemokratie entlarvt werden muss, um die revolutionären Bestrebungen wieder anzufachen, die sie vorübergehend erstickte. Deswegen richtet sich BECHERS Augenmerk nun auf den Kleinbürger, den ,deutsche[n] Michel“, der sich in diesem System eingerichtet hat und damit sein Überleben sichert. Dieser deutsche Michel, der „wie angetrunken“ vorübertorkelt, ist eine angesichts des neuen demokratischen Staats politikverdrossen gewordene Gestalt: ,Zuviel Parteiinteressen... Ich bin eben nun einmal kerndeutsch, damit soll man es bewenden lassen..."(GW VIII:146).

Die folgende „Darstellung des ,Deutschen Pfuhls““ (GW VIII:155) bietet eine Fortsetzung dieser grotesken Wiedergabe der Situation in der Weimarer Republik; das Deutschtum, das dort gefeiert wird, widerspricht selbstredend dem Internationalismus der kommunistischen Lehre (vgl. GW VIII:157). Der deutsche 
Michel ist bezeichnenderweise nicht nur von der Politik und der Aufforderung, sich selbst eine Meinung zu bilden, überfordert. Indem er sich zum „Mystiker“ erklärt (GW VIII:148), verkörpert er darüber hinaus eine Haltung, die nun im krassen Gegensatz zur Festspielfassung vom Drama konsequent als Irrweg ausgewiesen wird: die Suche nach Rettung in der Religion.

Die überarbeitete Fassung ist aber nicht nur in dieser Weise weltanschaulich ,korrigiert' und zugespitzt. BECHER bringt das Drama auch ästhetisch auf den neuesten Stand. Er ,kuriert‘ den „,von den üblichen expressionistischen Schauerkrämpfen durchschüttelte[n] Sprachkörper“ (GW VIII:104), der nun vor dem Hintergrund von Neuer Sachlichkeit und Dokumentarismus nicht mehr zeitgemäß erscheint, und setzt sich intensiver mit dem proletarischen Theater auseinander, das vor allem dank Erwin Piscator inzwischen stärker auf der deutsche Bühne etabliert ist. ${ }^{14}$ Während in der Festspielfassung von 1919 die Übergänge vom expressionistischen zum politischen Theater noch fließend sind, übernimmt BECHER jetzt die Forderung nach einem radikal neuen Ansatz, der konsequent mit dem traditionellen bürgerlichen Kunstverständnis bricht. In Levisite weist er die Erwartungen der Leserschaft an einen konventionellen Roman vehement zurück $;{ }^{15}$ in seiner „Bemerkung zur Umarbeitung“ zu Arbeiter Bauern Soldaten lehnt er es „mit aller Entschiedenheit ab, mit dieser Arbeit auch nur den Versuch gemacht zu haben, ein Drama im herkömmlichen Sinne zu schreiben“" (GW VIII:103).

Neben Musik legt BECHER den Einsatz von Scheinwerfern, Tafeln mit Inschriften und Zwischenbildern fest (GW VIII:155-159), fügt mit nun deutlich erhöhtem programmatischen Bewusstsein eine Reihe von Regieanweisungen zur Aufführungspraxis ein und erläutert seine Vorstellungen von der Inszenierung. So sollen zu Beginn ,revolutionäre Flugblätter“ verteilt werden, die Teilnehmer ,in Zügen geordnet durch die Stadt anmarschieren, mit Musik, roten Fahnen usw."; Berufsschauspieler seien ,nur insoweit zuzulassen, als sie sich bedingungslos dem Gesamtspiel unterordnen“ (GW VIII:103). Wo Piscator die „Trennungswand zwischen Bühne und Zuschauerraum“ auch bühnentechnisch einreißt (PISCATOR 1968:57), kennt BECHERS Konzept diese Grenzüberschreitung auf der Produktionsebene: Es neutralisiert die Differenz zwischen Künstler und Rezipient zugunsten einer „Gesamtschöpfung“ von Schauspielern und „mitwirkenden Massen“, die

14 Eine Übersicht der von Piscator inszenierten Stücke findet sich in INNES (1972:219f.). 
selbst „mit ihrer Phantasie, mit ihrem Darstellungsvermögen, mit ihrer Spannkraft“ zu beteiligen sind, sodass beide Gruppen „zu einer klassenbewußten organischen Einheit" zusammenwachsen (GW VIII:103).

Entsprechend baut BECHER in der zweiten Fassung von Arbeitern Bauern Soldaten die musikalischen Strukturen, die in der Erstfassung schon angelegt waren, merklich aus. Er arbeitet das Stück so um, dass es mit seinen nunmehr vier Teilen symphonischen Aufbau erhält, und lässt die Einzelstimmen zur gemeinsamen Motivwiederholung in einer Art und Weise zusammenfinden, wie in der Instrumentalmusik Soli einzelne Themen erarbeiten, bevor alle gemeinsam erklingen. ${ }^{16}$ Während in der Festspielfassung Prosa und Verse einander recht unsystematisch abwechseln, geht das Gespräch von Mann und Frau nun in dem Moment in die Versform über, in dem sich beide aus einem Mund ins Kollektiv eingliedern und im maschinenartigen Takt ,zu funktionieren“ haben: „Gib, daß alle Anforderungen, die an mich gestellt werden, ich erfülle. / Daß ich ein gut funktionierendes Glied sei / Im Mechanismus jener riesenhaften Kampfmaschine“ (GW VIII:125f.).

Im Gegensatz zu Piscators Ansatz freilich sind diese Anweisungen nicht auf die Etablierung eines neuen Theaters gerichtet, sondern dienen der Neubestimmung der (eigenen) Rolle als Dichter, deren Persönlichkeit sich nun dem gemeinsamen Kampf unterzuordnen hat. ${ }^{17}$ Dabei wird die neue Identität als Ergebnis einer Überwindung eines älteren bürgerlichen Dichtungskonzepts präsentiert, das in den Werken nun immer wieder Thema ist. Die „Gottesseuche“ im Kampfdrama Arbeiter Bauern Soldaten wird nicht nur ,produziert“ von „Philosophien“ und „religiöse[n] Systeme[n]“ (GW VIII:154), sondern auch von „Dichtern und Denkern“, die „Visionen“ haben und auf die „Normal-Menschen“ achselzuckend herabblicken (GW VIII:158). Der Dichter wird entsprechend dieser Skizze, die sowohl auf das bildungsbürgerliche deutsche Kulturgut als auch auf das Modell einer elitären Kunst etwa eines Stefan George verweist, wie es BECHER selbst noch im Juli 1920 angestrebt hatte, ${ }^{18}$ zum Agenten der Konterrevolution (GW VIII:141). Demgegenüber steht das Ideal eines Dichters, der in der Masse aufgeht und - wie BECHER in der Vorrede zum Kampfdrama - nur noch eine anteilige

16 Dies ist besonders deutlich an der Stelle, als zunächst ein Soldat, ein Bauer und ein Arbeiter auftreten, die dann gemeinsam die wichtigsten Schlagworte ihrer Soli wiederholen: „Für wen? / Warum? / Wozu?... / Genug. / Genug! / Genug!...“ (GW VIII:120).

17 Vgl. zu dieser Position bei Piscator Willett (1982:18).

18 Vgl. den Brief an Ludwig Meidner vom 3. Juli 1920 (BECHER 1993:90f.). 
Verfasserschaft beansprucht, die er sich mit den „mitwirkenden Massen“ und mit ,ihrer Phantasie, mit ihrem Darstellungsvermögen, mit ihrer Spannkraft" teilt (GW VIII:103).

In dieser Dichtermodellierung kehrt die Gegenüberstellung der zwei ,demokratischen' Systeme wieder, die die Grundanlage des Kampfdramas ausmachen. Der ,deutsche Dichter“, der 1919 seinen emphatischen „Gruß“ an ,Sowjet=Rußland" ausgerufen hatte, verkörpert nun den Irrweg religiöser Schwärmerei, der auch die Handlungsunfähigkeit des deutschen Michels, des Kleinbürgertums ausmacht. Dieses Denkmuster muss durch das Bekenntnis zum russischen Modell ersetzt werden, das nun das bürgerliche Element, den Individualismus und den ,Hang zum Mystischen` abwirft, um in der Anonymität der politischen Massenbewegung aufzugehen.

Dabei führt die Unterordnung des Dichters, der nicht mehr als geniales Individuum zu denken ist, das die Massen zum ,heiligen Land' führt, sondern nur als Teil einer größeren Bewegung revolutionäre Kunst hervorbringt, nun ausgerechnet zu einer nachhaltigeren autobiographischen Schwerpunktsetzung. Gerade als der Autor ,namenlos' werden soll, kommen die Gedichte wie authentische Kindheitserinnerungen daher (Im Schatten der Berge, 1928) und die Erzähltexte bevölkern sich mit autobiographisch inspirierten Protagonisten. Schon Peter Friedjung in Levisite ist eindeutig als Alter Ego BECHERS konzipiert und eröffnet den Reigen autobiographischer Fiktion, den er bis zu seinem Lebensende weiterführt. BECHER erzählt hier die Geschichte eines Bürgerlichen, der - aus dem Ersten Weltkrieg zurückgekehrt - sich nach und nach von seiner ,Herkunftsklasse" entfernt und vom deutschnationalen Patrioten zum Kommunisten wird. ${ }^{19}$ Ähnlich entwerfen auch die Gedichte aus dem Lyrikband Maschinenrhythmen (1926) einen Dichter, der in der Masse aufgeht - aber gerade deshalb umso mehr mit dem Namen des Autors verknüpft ist, weil dieses Konzept ein Überwindungsnarrativ voraussetzt, das diesen Namen explizit aufruft. Dort berichtet das lyrische Ich immer wieder von der eigenen Distanzierung von der religiösen Dichtung, die es vorher gepflegt hatte: „Darum alles / „Du' ist künftig: Namenlos, - Namenlos auch ist / Dieser Name: Becher Johannes R.“ (BECHER 1926b:95).

Das hat zur Folge, dass die dichterischen Arbeiten BECHERS nicht nur dadurch eine kommunistische Weltrevolution auf deutschem Boden in Gang zu setzen beabsichtigen, indem sie sich thematisch diesen Fragen zuwenden und die Mittel des russischen Massentheaters als Agitation aufgreifen. Viel wichtiger ist, dass sie als Zeugnisse der biographischen Entwicklung ihres Autors fungieren, der

Deshalb lagen psychologische Lektüren der Romane nahe, z. B. RoHRWASSER (1980). 
gerade durch seine Überarbeitungspraxis - stets mit entsprechenden programmatischen Vorworten begleitet wird - eine Vorbildfigur abgibt, was über die rein fiktionale Wirkung der literarischen Texte und selbst die starke (potentielle) performatorische Komponente der Theaterstücke bei weitem hinausgeht.

Die Aufspaltung der Dichterfigur in den bürgerlichen Visionär einerseits und die „Rote Nachtigall“ (BECHER 1926b:11) andererseits reagiert auf ein persönliches und dann doch wieder verallgemeinerbares Problem. Die „Künstler, die [...] aus dem bürgerlichen Lager stammen und die [...] frei gewählt haben, auf der Seite des revolutionären Proletariats den Entscheidungskampf zwischen Kapital und Arbeit mit auszukämpfen“ (GW VIII:103), haben sich nun von allem zu distanzieren, was mit ihrer Herkunftsklasse zusammenhängt - und das hat vor allem ästhetische Konsequenzen: BECHER, der schon als Expressionist eine gewisse Antibürgerlichkeit kultivierte, macht sich die Klassenkampfrhetorik zu eigen und erzählt die eigene Kindheit und Jugend nun mehrfach als Emanzipation von den Werten der bürgerlichen Herkunftsfamilie, die vor allem der Vater in den Romanen Levisite und Abschied repräsentiert. ${ }^{20}$ Der „,deutsche Dichter“, der schon 1919 die „Russische Sowjet-Republik“ grüßt und 1924 ein „HeldenGedicht vom Heldentum derer, / Die Tag und Nacht, / Nacht und Tag die Parteifragen diskutieren", schreibt (GW VIII:189), ist nun klar identifizierbar als jener „Becher, Johannes R.“ aus der „Maschinen-Zeit“ (BECHER 1926b:95), weil erst diese Rückführung auf eine konkrete Person den Beweis für die Wirklichkeitsrelevanz erbringt und die Überzeugungskraft der kommunistischen Lehre sichert.

Während die an das Modell des poeta vates angelehnte Konzeption von 1919 den Dichter nur als bloßen Verkünder des ,heiligen Reichs‘ kennt, das die Weltrevolution herbeiführen wird, führt die autobiographische Rückbindung des Dichtermodells ab 1923/1924 zu einer Situation, in der BECHER mit seiner Person für die kommunistische Sache ,bürgt‘. Der unwahrscheinliche Weg des Bürgerlichen, der sich zum Klassenkampf bekennt, soll Vorbildwirkung für alle Dichter bürgerlicher Herkunft entfalten, die jetzt den Fehler machen, auf der Seite der Wahrheitsverschleierung zu stehen. ${ }^{21}$ Für alle anderen ist er ein Beispiel dafür, dass eine Entwicklung bereits in Gang gekommen und die zunehmende Einsicht auch der bürgerlichen Intellektuellen nicht mehr aufzuhalten

\footnotetext{
${ }^{20}$ Vgl. unter dieser Perspektive vor allem RoHRWASSER (1980).

${ }^{21}$ Vgl. zu diesem Kontext in Levisite WitTMANN (1980:33).
} 
ist. Die Rolle des Dichters JOHANNeS R. BECHER ist daher gleichermaßen Aufforderung zum Anschluss an die Bewegung und Exempel dafür, dass sie stattfindet.

\section{Literatur}

Anonymus: Proletarisches Theater (17.10.1920). In: MANFREd BraunECK (ed.): Die rote Fahne. Kritik, Theorie, Feuilleton 1918-1933. München (=UTB für Wissenschaft Uni-Taschenbücher Literaturwissenschaft 127), 92-95.

Becher, Johannes R. (1914): Verfall und Triumph. Erster Teil: Gedichte. Berlin.

Becher, Johannes R. (1919): An Alle! Neue Gedichte. Berlin-Wilmersdorf.

BECHER, JOHANNES R. (1919): Gedichte um Lotte. Leipzig.

BeCHER, JohANNES R. (1920): Ewig im Aufruhr. Berlin.

BeCher, Johannes R. (1920): Zion. Gedichte. München.

BECHER, JohANNES R. (1921): Um Gott. Leipzig.

BECHER, JOHANNES R. (1922): Verklärung. Berlin.

Becher, Johannes R. (1924): Am Grabe Lenins. Wien.

BECHER, JOHANNES R. (1926a): $(\mathrm{CHCl}=\mathrm{CH})_{3} A s$ (Levisite) oder Der einzig gerechte Krieg. Roman. Wien.

BECHER, JOHANNES R. (1926b): Maschinenrhythmen. Berlin.

BECHER, JohANNES R. (1959): Widmungsblatt an die russische Revolution 1917 [Faksimile der Handschrift]. In: Sinn und Form. Zweites Sonderheft Johannes R. Becher, 116.

BeCher, Johannes R. (1993): Briefe 1909-1958, ed. HARder, Rolf. Berlin / Weimar.

BeCKer, Sabina (2009): Autorschaft vs. Dichtertum. Moderne-Konzepte bei Brecht und Benn. In: ACHIM Aurnhammer, Werner Frick, GÜNTER SAßE (eds.): Gottfried Benn - Bertolt Brecht. Das Janusgesicht der Moderne. Würzburg, 255-271.

Behrens, AleXANDER (2003): Johannes R. Becher. Köln.

FÄHNDERS, WALTER (2010²): Avantgarde und Moderne 1890-1933. Lehrbuch Germanistik, 2. Stuttgart.

Hagelweide, Gert (1968): Das publizistische Erscheinungsbild des Menschen im kommunistischen Lied. Eine Untersuchung der Liedpublizistik der KPD (1919-1933) und der SED (1945-1960). Bremen.

HARTUNG, GÜNTER (2002): Bechers Festspiel „Arbeiter Bauern Soldaten “ von 1919. In: HARTUNG, GÜNTER (ed.): Literatur und Welt. Vorträge. Leipzig, 283-292.

HOPSTER, NORBERT (1969): Das Frühwerk Johannes R. Bechers. Bonn.

InNes, Christopher D. (1972): Erwin Piscator's Political Theatre. The Development of Modern German Drama. Cambridge. 
KIESEL, HeLmUTH (2017): Geschichte der deutschsprachigen Literatur von 1918 bis 1933, München (=Geschichte der deutschen Literatur 10).

NÖSSIG, MANFRED / ROSENBERG, JOHANNA / SCHRADER, BÄRBEL (1980): Literaturdebatten in der Weimarer Republik. Zur Entwicklung des marxistischen literaturtheoretischen Denkens 1918-1933. Berlin / Weimar.

PAtTerson, Michael (1981): The Revolution in German Theatre 1900-1933. New York. PISCATOR, ERWIN (1968): Schriften II: Aufsätze, Reden, Gespräche, ed. HofFMANN, LuDWIG. Berlin.

REUS, GUNTER (1978): Oktoberrevolution und Sowjetrußland auf dem deutschen Theater. Zur Verwendung eines geschichtlichen Motivs im deutschen Schauspiel von 1918 bis zur Gegenwart. Bonn, 131-144.

Rohrwasser, Michael (1980): Der Weg nach oben. Johannes R. Becher, Politiken des Schreibens. Basel.

RYkLIN, MiнAiL K. (2008): Kommunismus als Religion. Die Intellektuellen und die Oktoberrevolution. Frankfurt a. M. u.a.

Schlenstedt, Silvia (1976): Wegscheiden. Deutsche Lyrik im Entscheidungsfeld der Revolutionen von 1917 und 1918. Berlin, 14-52, 113-158.

Walter, HANs-AlBerT (1978): Deutsche Exilliteratur 1933-1950. Bd. 1.1: Die Mentalität der Weimardeutschen/Die „Politisierung “ der Intellektuellen. Stuttgart.

WiLlett, John (1982): Erwin Piscator. Die Eröffnung des politischen Zeitalters auf dem Theater. Aus dem Englischen von Peter Keller. Frankfurt a. M. (=Edition Suhrkamp 924).

WitTMAnN, Livia Z (1980): „Zur Funktion des Dichters und Intellektuellen im Roman $\left(\mathrm{CHCL}=\mathrm{CH}_{3}\right)$ As (Levisite) oder Der einzig gerechte Krieg“. In: Seminar 16:26-36.

WOLFF, HANNELORE (1985): Volksabstimmung auf der Bühne? Das Massentheater als Mittel politischer Agitation. Frankfurt a. M. u.a (=Europäische Hochschulschriften, Reihe 30, Theater-, Film- und Fernsehwissenschaften 23). 